\title{
Disrupting feedback processing interferes with rule-based but not information-integration category learning
}

\author{
W. TODD MADDOX \\ University of Texas, Austin, Texas \\ F. GREGORY ASHBY \\ University of California, Santa Barbara, California \\ A. DAVID ING \\ University of Texas, Austin, Texas \\ and \\ ALAN D. PICKERING \\ Goldsmiths College, University of London, London, England
}

\begin{abstract}
The effect of a sequentially presented memory scanning task on rule-based and informationintegration category learning was investigated. On each trial in the short feedback-processing time condition, memory scanning immediately followed categorization. On each trial in the long feedbackprocessing time condition, categorization was followed by a 2.5-sec delay and then memory scanning. In the control condition, no memory scanning was required. Rule-based category learning was significantly worse in the short feedback-processing time condition than in the long feedback-processing time condition or control condition, whereas information-integration category learning was equivalent across conditions. In the rule-based condition, a smaller proportion of observers learned the task in the short feedback-processing time condition, and those who learned took longer to reach the performance criterion than did those in the long feedback-processing time or control condition. No differences were observed in the information integration task. These results provide support for a multiple-systems approach to category learning and argue against the validity of single-system approaches.
\end{abstract}

The ability to categorize is critical to the survival of all organisms (Ashby \& Maddox, 1998). Every day, organisms make thousands of categorization judgments and are often remarkably accurate. A growing body of research suggests that, in humans, the learning of different types of category structures is mediated by different categorization and memory systems (e.g., Ashby \& Ell, 2001; Erickson \& Kruschke, 1998; Pickering, 1997; Reber \& Squire, 1994; Smith, Patalano, \& Jonides, 1998; however, see Nosofsky \& Johansen, 2000). Most multiple-systems theorists agree that one system is explicit and at least one is implicit. For example, the competition between verbal and implicit systems (COVIS) model assumes an explicit

This research was supported in part by National Institutes of Health Grant R01 MH59196 to W.T.M., Public Health Service Grant MH3760 to F.G.A., and a McDonnell-Pew Consortium grant. We thank Maribel Chavez, Kelli Hejl, Randy Jupio, and Jessica Valverde for help with data collection, and three anonymous reviewers for helpful comments on an earlier version of the manuscript. Correspondence concerning this article should be addressed to W. T. Maddox, University of Texas, 1 University Station A8000, Department of Psychology, Austin, TX 78712 (e-mail: maddox@psy.utexas.edu). hypothesis-testing system and an implicit procedural learning system (Ashby, Alfonso-Reese, Turken, \& Waldron, 1998). The explicit hypothesis-testing system is assumed to dominate the learning of rule-based category learning tasks, whereas the implicit procedural learning system dominates learning of information-integration category learning tasks.

Rule-based category learning tasks are those in which the category structures can be learned via some explicit reasoning process. Frequently, the rule that maximizes accuracy (i.e., the optimal rule) is easy to describe verbally (Ashby et al., 1998). In many applications, only one stimulus dimension is relevant, and the observer's task is to discover this relevant dimension and then map the different dimensional values to the relevant categories. In other applications, two dimensions are relevant, and the observer's task is to learn a decision criterion along each dimension and map the different dimensional values to the relevant categories. For example, if the stimulus is a sine wave grating that varies in spatial frequency and spatial orientation, the observer might set one criterion along spatial frequency to determine whether the stimulus is of "low" or "high" frequency and one along spatial 
orientation to determine whether the stimulus is of "shallow" or "steep" angle. The decision along each dimension might then be integrated to determine category membership (e.g., low frequency/shallow angle and high frequency/steep angle stimuli are assigned to Category A). This integration is postdecisional, because a decision is first made about the value along each dimension and then that information is integrated to generate a response.

Information-integration category learning tasks, on the other hand, are those in which accuracy is maximized only if information from two or more stimulus components (or dimensions) is integrated at some predecisional stage (Ashby \& Gott, 1988). Perceptual integration could take many forms - from treating the stimulus as a gestalt to computing a weighted linear combination of the dimensional values. In many cases, the optimal rule in information integration tasks is difficult or impossible to describe verbally (Ashby et al., 1998). In contrast to information integration rules, a conjunctive rule (e.g., "Respond $\mathrm{A}$ if the stimulus is small on dimension $x$ and small on dimension $y$ ") is one in which the observer applies separate decisions about each dimension (e.g., small or large) and then combines the outcome of these decisions when making his or her categorization decision (i.e., integration is not predecisional). Such rules can be applied to information integration conditions, but they generally lead to suboptimal levels of accuracy. Unlike information integration rules, conjunctive rules are easy to verbalize.

COVIS assumes that learning in rule-based tasks is dominated by an explicit system in which working memory and executive attention are used. This system learns through a conscious process of hypothesis generation and testing. On each trial, the observer selects a response by using an explicit rule or hypothesis. If the feedback signals that the response was correct, the same rule is used again on the next trial. However, if the feedback indicates that the response was incorrect, then the observer must decide whether to try the same rule again or switch to a new rule. If the latter decision is made, then a new rule must be selected and, in addition, attention must be switched from the old rule to the new. These operations require time and attention. In contrast, learning in information integration tasks is assumed to be dominated by an implicit, procedural, learning-based system that depends on a reward signal to strengthen the appropriate (stimulus category) associations in a relatively automatic fashion (Ashby et al., 1998; Ashby \& Ell, 2001). Thus, in COVIS feedback processing in the two systems is very different. In particular, feedback processing requires time and attention in the explicit system, but it occurs almost automatically in the procedural learning system. This article provides the first known test of this prediction.

In the next section, we briefly outline the neurobiological underpinnings of the proposed explicit and procedural learning systems and review previous related work. We then outline the new experimental manipulation, present the results from an experiment that introduces this manipulation, and summarize the results.

\section{Previous Tests of a Neurobiological Model of Category Learning}

A detailed discussion of the neurobiology assumed by COVIS is available in numerous articles (e.g., Ashby \& Ell, 2001); here, only a brief overview will be offered. COVIS assumes that learning in rule-based tasks is dominated by an explicit system that learns through a conscious process of hypothesis generation and testing. In contrast, learning in information integration tasks is dominated by a procedural learning-based system that is mediated largely within the tail of the caudate nucleus (Ashby et al., 1998; Ashby \& Ell, 2001). A dopamine-mediated reward signal is critical for learning in this system. The idea is that an unexpected reward causes dopamine to be released from the substantia nigra into the tail of the caudate nucleus and that the presence of this dopamine strengthens recently active synapses (see, e.g., Schultz, 1992; Wickens, 1993).

The rule-based category learning system is under conscious control and has full access to working memory and executive attention. As a result, the placement and timing of the feedback signal should have little effect on rule-based category learning because this information can be held consciously in working memory. In contrast, a procedural learning system that is mediated within the tail of the caudate nucleus would not be accessible to conscious awareness and is far removed from working memory. As a result, it would depend more heavily on local learning mechanisms that are likely associated with stimulus-response association learning. This system should learn better with feedback as opposed to observational training, and with immediate as opposed to delayed feedback. Ashby, Maddox, and Bohil (2002) compared rule-based and information-integration category learning across observational and feedback training conditions, and Maddox, Ashby, and Bohil (2003) compared rule-based and information-integration category learning across immediate and delayed feedback conditions. In line with predictions from COVIS, observational training and delayed feedback were as effective as feedback training and immediate feedback with rule-based categories, but with information integration categories observational training and delayed feedback led to significant decreases in category learning performance. The procedural learning system is associated with motor performance (see, e.g., Hazeltine \& Ivry, 2001; Willingham, 1998; Willingham, Nissen, \& Bullemer, 1989), and thus should be affected by changes in the response requirements. As was suggested by Willingham, et al.'s (1989) work, the procedural learning system should learn better in situations in which each category is associated with a fixed response location. Ashby, Ell, and Waldron (2003; see also Maddox, Bohil, \& Ing, in press) found support for this prediction. All of these studies dissociate rule-based from information-integration category learning by adversely affecting information-integration but not rule-based category learning. Several studies have established dissociations in the opposite direction. First, Waldron and Ashby (2001) showed that a rule-based category learning task 
was disrupted more by a simultaneous (numerical Stroop) task than was an information integration task. Similarly, Ashby, Noble, Filoteo, Waldron, and Ell (2003) found that Parkinson's disease patients were much more impaired at rule-based category learning than at informationintegration category learning.

Collectively, these studies provide powerful evidence that human category learning is mediated by multiple systems. A more ambitious goal than establishing that there are multiple category learning systems, however, is to investigate the qualitative properties of the component systems. In this article, we address this more ambitious goal. Although the results presented here provide yet more support for the multiple systems hypothesis, to our mind they provide, more importantly, the first investigation of the nature of feedback processing in the component systems.

\section{Overview of the Study}

Recall that the explicit hypothesis-testing system has full access to working memory and executive attention and learns through an active process of hypothesis generation and testing. This system is very flexible, being unaffected by the nature and timing of feedback and by changes in the motor aspects of the response. In the procedural learning system, on the other hand, learning is strongly affected by the nature and timing of feedback and by changes in the motor aspects of the response. The increased flexibility of the explicit hypothesis-testing system, however, comes at the extra cost of working memory and attentional capacity. For example, Ashby et al. (1998) hypothesized that following feedback that an error has just occurred, the explicit system initiates the following sequence of events: (1) Update (i.e., decrease) the salience of the current categorization rule; (2) if the salience of the current rule has dropped sufficiently, then identify a new plausible candidate rule; and (3) switch attention from the old rule to the new rule. As was mentioned above, this sequence requires both attention and time. On the other hand, the procedural learning system processes the feedback more or less automatically. If these hypotheses are correct, then it should be possible to interfere with rule-based category learning if, immediately after the feedback is given, observers are asked to perform a second, unrelated task that requires working memory and attention. The idea is that the performance

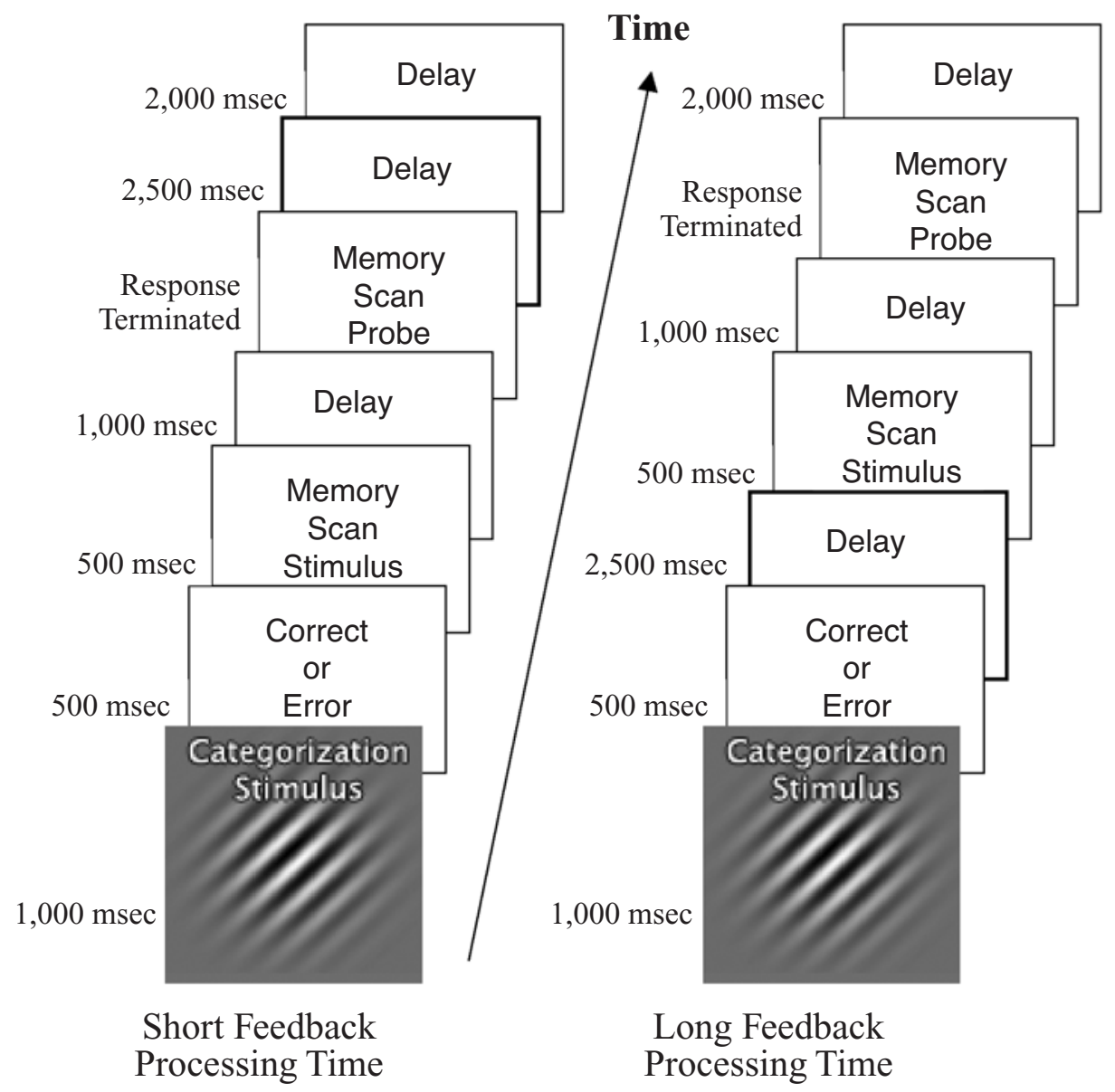

Figure 1. Basic design of the short and long feedback-processing time conditions. 
of this task will prevent normal feedback processing in the explicit system, but it will have little or no effect on feedback processing in the procedural learning system.

As the second task, we chose four-item memory scanning. In the short feedback-processing time condition, the memory scanning task directly followed the corrective feedback associated with category learning. In the long feedback-processing time condition, a short delay preceded the memory scanning task. On each trial, the observer was to perform two tasks sequentially. First, a categorization task was performed using traditional immediate feedback training. Following completion of the categorization trial, the observer performed a four-item memory scanning task. The timing of a typical short and a long feedback-processing time trial is depicted in Figure 1. On each trial in the long feedback-processing time condition, the observer was shown a categorization stimulus for $1,000 \mathrm{msec}$, generated a categorization response, and received $500 \mathrm{msec}$ of corrective feedback. A 2,500msec blank screen delay followed the categorization trial, and then four randomly sampled (without replacement) digits between 0 and 9 were displayed for $500 \mathrm{msec}$, followed by a 1,000-msec blank screen. A single probe digit was then displayed, and the observer had to decide whether the probe item was or was not in the memory set. Following the memory scanning response was a short intertrial interval (ITI) and the initiation of the next trial. On each trial in the short feedback-processing time condition, the categorization procedure was identical to that of the long feedback-processing time condition; however, instead of the categorization task's being followed by a $2,500-\mathrm{msec}$ blank screen delay, the memory scanning task was initiated immediately, and the 2,500-msec blank screen delay followed completion of the memory scanning task.

The rule-based and information-integration category structures used in the present study are described in Figure 2 . In the rule-based task (Figure 2A), the optimal bound requires observers to attend to spatial frequency and ignore orientation. The stimulus dimensions of Gabor patches are separable, have simple verbal labels (bar width and orientation), and have no emergent (or configural) features (e.g., all patches are exactly the same size and shape, regardless of frequency or orientation). For these reasons, there is a simple explicit rule that separates the contrasting categories. In particular, the vertical bound in Figure 2A corresponds to the rule, "Respond A if the bars are thick and B if they are thin." In the information integration task (Figure $2 \mathrm{~B}$ ), which was generated by rotating the rule-based categories by $45^{\circ}$ and shifting each stimulus away from the center of the spatial frequency-spatial orientation space, equal attention must be allocated to both stimulus dimensions. In this task, there is no simple verbal description of the optimal decision bound. The category discriminability $\left(d^{\prime}\right)$ and distribution parameters are outlined in Table 1 . The rule-based $d^{\prime}=3.5$, whereas the information integration $d^{\prime}=9.2$. These $d^{\prime}$ values were chosen because they yielded equivalent category learning in a control condition that excluded the memory scanning task. These data are outlined in Experiment $1 \mathrm{~A}$, to which we now turn.

\section{EXPERIMENT 1A}

When testing the hypothesis that short feedbackprocessing times should differentially affect rule-based but not information-integration category learning, it is critical to ensure that rule-based and information-integration category learning profiles are equivalent under control conditions that exclude the memory scanning task. If control condition performance is not equated, then task difficulty differences might be used to explain any differential effects of the long versus short processing time manipulation. To determine whether the rule-based and information-integration category structures presented in Figure 2 yielded equivalent category learning profiles, we collected data from participants in a control condition.

\section{Method}

\section{Observers and Design}

Fifty observers were solicited from the University of Texas community and received course credit for their participation. Half were randomly assigned to the rule-based condition and half to the information integration condition. No observer participated in more than one experimental condition. All of the observers were tested for $20 / 20$ vision using a Snelling eye chart. Each observer completed one session of approximately $60 \mathrm{~min}$ in duration.

\section{Stimuli and Stimulus Generation}

In this experiment, we used the randomization technique introduced by Ashby and Gott (1988). Forty Category A and 40 Category B stimuli from the rule-based categories were generated by sampling randomly from two bivariate normal distributions. The stimuli for the information integration categories were generated by rotating the 80 rule-based stimuli clockwise by $45^{\circ}$ and then shifting the spatial frequency and spatial orientation by an amount that resulted in the appropriate $d^{\prime}$ (see Table 1). Each set of 80 stimuli was displayed in a random order in each of four blocks of trials.

The stimuli were computer generated and displayed on a 21-in. monitor with $1,360 \times 1,024$ resolution in a dimly lit room. Each Gabor patch was generated using Matlab routines from Brainard's (1997) Psychophysics Toolbox. Each random sample $\left(x_{1}, x_{2}\right)$ was converted to a stimulus by deriving the frequency, $f=.25+\left(x_{1} / 50\right)$, and orientation, $o=x_{2}(\pi / 500)$. For example, the Category A mean

Table 1

Category Distribution Parameters for the Information-Integration and Rule-Based Category Structures

\begin{tabular}{|c|c|c|c|c|c|c|c|c|c|c|c|}
\hline \multirow[b]{2}{*}{ Condition } & \multicolumn{6}{|c|}{ Category A } & \multicolumn{5}{|c|}{ Category B } \\
\hline & $d^{\prime}$ & $\mu_{f}$ & $\mu_{o}$ & $\sigma_{f}^{2}$ & $\sigma_{o}^{2}$ & $\operatorname{cov}_{f, o}$ & $\mu_{f}$ & $\mu_{o}$ & $\sigma_{f}^{2}$ & $\sigma_{o}^{2}$ & $\operatorname{cov}_{f, o}$ \\
\hline Information integration & 9.2 & 272 & 153 & 4,538 & 4,538 & 4,463 & 327 & 97 & 4,538 & 4,538 & 4,463 \\
\hline Rule based & 3.5 & 285 & 125 & 75 & 9,000 & 0 & 315 & 125 & 75 & 9,000 & 0 \\
\hline
\end{tabular}

Note-cov, covariance; $f$, frequency; $o$, orientation. 
$\mathbf{A}$

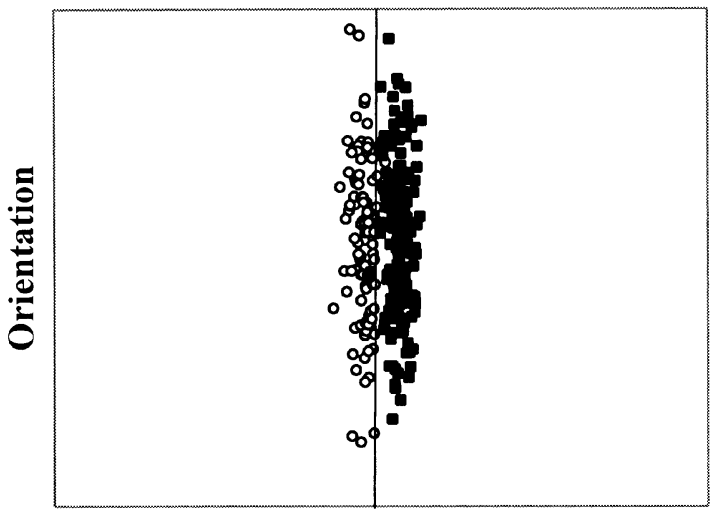

Frequency

B

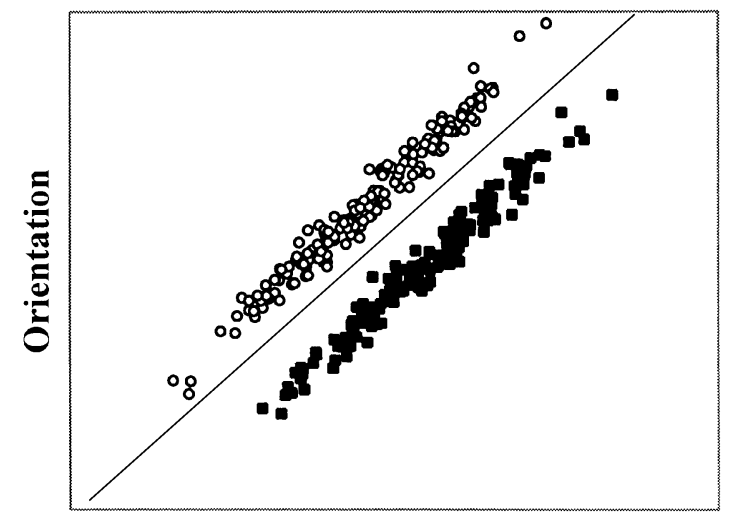

Frequency

Figure 2. (A) Rule-based and (B) information-integration category structures. Each open circle denotes the spatial frequency and spatial orientation of a single Gabor pattern from Category A. Each filled square denotes the spatial frequency and spatial orientation of a single Gabor pattern from Category $B$. The solid line in each panel denotes the location of the optimal decision bound.

for the rule-based (RB)-9.2 category structure was converted to a Gabor pattern with $f=.25+(260 / 50)=5.45 \mathrm{cpd}$ and $o=$ $125(\pi / 500)=0.785$ radians counterclockwise from horizontal. The scaling factors were chosen in an attempt to equate the salience of frequency and that of orientation.

\section{Procedure}

The observers were informed that there were two categories and that each category was equally likely. They were informed that perfect performance was possible and were instructed to learn about the two categories. They were told to be as accurate as possible and not to worry about speed of responding. On each trial, a stimulus was presented until the participant pressed either the A key or the B key; corrective feedback was presented for $500 \mathrm{msec}$ and followed by a 2,500-msec ITI.

\section{Results and Discussion}

A 2 (category structure: rule based vs. information integration) $\times 4$ (blocks) mixed design ANOVA was conducted on the accuracy rates. The accuracy rates averaged across observers are displayed in Figure 3A. The main ef- fect of block was significant $\left[F(3,144)=29.83, M S_{\mathrm{e}}=\right.$ $.0056, p<.001]$, suggesting improved performance with experience. Most importantly, the effect of category structure $(F<1)$ and the interaction $[F(3,144)=1.95$, $\left.M S_{\mathrm{e}}=.0056, p>.10\right]$ were both nonsignificant. The latter finding verifies that performance in the rule-based and information integration conditions are equivalent and, thus, that any performance differences observed in the feedback-processing time conditions cannot be attributed to a priori differences in rule-based and informationintegration category learning difficulty.

\section{EXPERIMENT 1B}

In Experiment 1B, participants were asked to learn the rule-based or information-integration category structures presented in Figure 2 under short or long feedbackprocessing time conditions.

\section{Method}

\section{Observers and Design}

One hundred five observers were solicited from the University of Texas community and received course credit for their participation. Twenty-six and 29 observers participated in the rule-based task under long and short feedback-processing time conditions, respectively. Twenty-five observers participated in the information integration task under the long feedback-processing time condition, and 25 participated in that task under the short-feedback condition. No observer participated in more than one experimental condition. All observers were tested for 20/20 vision using a Snelling eye chart. Each observer completed one session of approximately $60 \mathrm{~min}$ in duration.

\section{Stimuli and Stimulus Generation}

Category learning. The category learning stimuli and stimulus generation procedures were identical to those outlined in Experiment $1 \mathrm{~A}$.

Memory scanning. On each trial, four digits were sampled randomly (without replacement) from the set of single-digit numbers from 0 to 9 . The four selected digits were displayed for $500 \mathrm{msec}$ in 48-point type in a horizontal array, separated from each other by 100 pixels, and vertically centered on the screen. A blank screen was then displayed for $1,000 \mathrm{msec}$. Next, a single digit was sampled randomly with a .5 probability of being sampled from the memory set. The selected digit was centered on the screen along with the question, "Was this item in the memory set?" The observer then responded "yes" or "no" by pressing one of two keys that were different from those used for category learning.

\section{Procedure}

The procedure was identical to that of Experiment 1A with the following additions. For memory scanning, the observers were informed that high levels of performance were possible and that they should respond as quickly and accurately as possible. If performance in the memory scanning task was below $90 \%$ accuracy at the end of any trial, the observers were told to increase their memory scanning accuracy. These notifications stopped once memory scanning accuracy was above $90 \%$. The procedure for a trial in the long and short feedback-processing time conditions is outlined in Figure 1.

\section{Results}

\section{Memory Scanning Performance}

Accuracy. The participants performed the memory scanning task with high accuracy, achieving an overall 


\section{(A)}

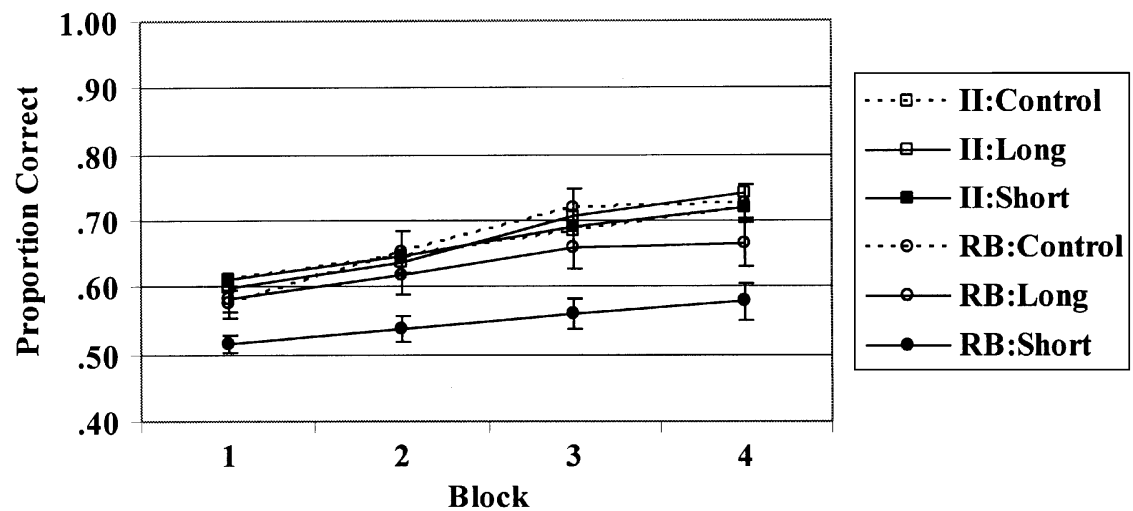

(B)

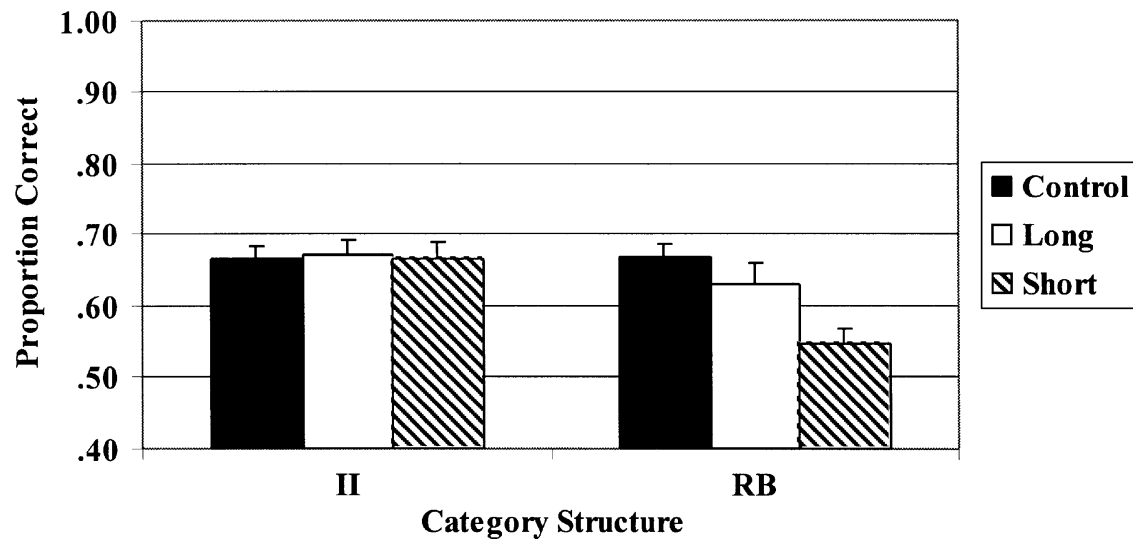

Figure 3. (A) Proportion correct for the rule-based (RB, circles) and information-integration (II, squares) category structures under control and long and short feedback-processing conditions for each of the four blocks of trials (standard error bars are included). The broken lines denote the control condition data, and the solid lines the long and short feedback-processing time condition data. (B) Proportion correct for the same conditions averaged over blocks (standard error bars are included).

accuracy level of $97.6 \%$. There was no significant difference in memory scanning accuracy in the rule-based category learning task across the long $(98.1 \%)$ and short $(97.3 \%)$ feedback-processing time conditions $[t(53)=$ $1.60, p>.10]$, nor was there a significant difference in accuracy in the information-integration category learning task across the long (97.5\%) and short (97.6\%) feedbackprocessing time conditions $[t(48)=.17, p>.50]$. In addition, there was no significant difference in memory scanning accuracy in the long feedback-processing time condition across the rule-based and information integration tasks $[t(49)=1.08, p>.20]$, nor was there a significant difference in memory scanning accuracy in the short feedback-processing time condition across the rule-based and information integration tasks $[t(52)=.65, p>.50]$.

Mean correct response time (RT). Mean correct RT in the memory scanning task was $1,484 \mathrm{msec}$. There was no significant difference in mean memory scanning RT in the rule-based category learning task across the long $(1,417 \mathrm{msec})$ and short $(1,502 \mathrm{msec})$ feedback-processing time conditions $[t(53)=.60, p>.50]$, nor was there a significant difference in mean memory scanning RT in the information-integration category-learning task across the long $(1,474 \mathrm{msec})$ and short $(1,542 \mathrm{msec})$ feedbackprocessing time conditions $[t(48)=.44, p>.60]$. In addition, there was no significant difference in mean memory scanning RT in the long feedback-processing time condition across the rule-based and information integration tasks $[t(49)=.39, p>.60]$, nor was there a significant difference in mean memory scanning RT in the short feedback-processing time condition across the rule-based and information integration tasks $[t(52)=.27, p>.70]$. In summary, mean memory scanning accuracy and correct response RTs were not significantly different in any of the four experimental conditions (i.e., 2 category structures $\times 2$ feedback-processing time conditions).

\section{Category Learning Performance}

For completeness, we included the data from Experiment $1 \mathrm{~A}$ in these analyses. A 3 (condition: control vs. long 
vs. short feedback-processing time) $\times 2$ (category structure: rule based vs. information integration) $\times 4$ (blocks) mixed design ANOVA was conducted on the accuracy rates. The accuracy rates averaged across observers are displayed in Figure 3A. The effects of category structure $\left[F(1,149)=8.61, M S_{\mathrm{e}}=.048, p<.01\right]$, condition $\left[F(2,149)=4.17, M S_{\mathrm{e}}=.048, p<.05\right]$, and block $\left[F(3,447)=64.49, M S_{\mathrm{e}}=.0058, p<.001\right]$ were all significant. The category structure and condition effects were qualified by a significant interaction $[F(2,149)=$ $\left.4.10, M S_{\mathrm{e}}=.048, p<.05\right]$. To determine the locus of this interaction, we repeated the analyses separately for the information-integration and rule-based category structures. The relevant data are plotted in Figure 3B.

For the information-integration category structure data, the only effect that reached significance was the block effect $\left[F(3,216)=47.04, M S_{\mathrm{e}}=.0046, p<.001\right]$. The condition effect and the interaction both yielded $F$ s of less than 1 . For the rule-based category structure data, the main effects of condition $\left[F(2,77)=7.68, M S_{\mathrm{e}}=.054\right.$, $p<.001]$ and block $\left[F(3,231)=23.64, M S_{\mathrm{e}}=.007, p<\right.$ $.001]$ were significant, and the interaction was marginally significant $\left[F(6,231)=2.07, M S_{\mathrm{e}}=.007, p=.06\right]$. To determine the locus of the condition effect, we compared (1) performance in the control condition with that in the long feedback-processing time condition, (2) performance in the long feedback-processing time condition with that in the short feedback-processing time condition, and (3) performance in the control condition with that in the short feedback-processing time condition. The results were clear: Performance did not differ between the control condition $(66.8 \%)$ and the long feedbackprocessing time condition $[63.1 \% ; t(49)=1.05, p=$ $.30]$, but performance did differ between the long (63.1\%) and short $(54.8 \%)$ feedback-processing time conditions $[t(53)=2.66, p<.01]$ and between the control and short feedback-processing time conditions $[t(52)=4.13, p<$ $.001]$.

These results provide strong support for the a priori prediction of COVIS (Ashby et al., 1998). Informationintegration category learning was equivalent across the control, long, and short feedback-processing time conditions. However, the short feedback-processing time condition led to a significant performance decrease of $8 \%$ relative to the long feedback-processing time condition for the rule-based task, and a decrease of $12 \%$ relative to the control condition. These results support the claim that rulebased category learning requires working memory and attention to process the feedback, whereas informationintegration category learning is mediated by a procedural learning-based system in which the feedback is processed automatically.

Although the results provide further evidence of a multiple-systems approach to category learning, a detailed examination of the data from each observer suggested that many observers in the rule-based conditions failed to respond above chance (50\%). In fact, $31 \%$ and $41 \%$ of the observers in the rule-based condition, in the long and short feedback-processing time conditions, respectively, did not exceed chance performance during their final block of trials, whereas only $8 \%$ of the observers in the information integration condition, across both the long and short feedback-processing time conditions, did not exceed chance performance during their final block of trials. Importantly, the percentage of observers who failed to respond above chance during their final block of trials was low and nearly equivalent in the control conditions (rule based $=12 \%$; information integration $=8 \%$ ). These findings suggest that the accuracy results presented above include a mixture of observers who learned something about the categories and observers who were responding essentially randomly, and that this mixture was affected by the short/long feedbackprocessing time manipulation in the rule-based condition but not in the information integration condition. In light of this fact, we reanalyzed the data using a proportion of learners/trials-to-criterion approach that is commonly used under these conditions (see, e.g., Ashby, Noble, et al., 2003; Waldron \& Ashby, 2001). We turn now to these analyses.

\section{Proportion of Learners/Trials to Criterion}

To determine whether each observer learned the categorization problem, we took the following approach. We defined an 80-trial window on Trials 1-80. We then computed the observer's accuracy over these 80 trials and determined whether the observer's proportion correct was greater than or equal to .65. If it was, the observer was classified as a learner and the trials to criterion was set at 80 trials. If it was not, we dropped Trial 1 and added Trial 81 (i.e., the window included Trials 2-81) and computed accuracy again. If the observer's proportion correct was greater than or equal to .65 , then the observer was classified as a learner with trials to criterion of 81 . When an observer's accuracy did not exceed .65 , we continued this process until it met the .65 performance criterion or until we reached the last trial of the study (Trial 320). If the observer never met the criterion, then he or she was classified as a nonlearner.

The proportion of rule-based and information integration learners in the control, long, and short feedbackprocessing time conditions are displayed in Figure 4A. $\chi^{2}$ tests were conducted on the learner/nonlearner proportions for the control, long, and short feedbackprocessing time conditions separately for the rule-based and information-integration category structures. A 3 (condition) $\times 2$ (learner/nonlearner) $\chi^{2}$ test on the rulebased condition data was significant $\left[\chi^{2}(2)=11.64, p<\right.$ $.01]$. Follow-up tests suggested that there were significantly fewer rule-based learners in the short feedbackprocessing time condition than in either the long feedbackprocessing time condition $\left[\chi^{2}(1)=4.03, p<.05\right]$ or the control condition $\left[\chi^{2}(1)=11.27, p<.001\right]$, but that the proportion of learners did not differ across the control and long feedback-processing time conditions $\left[\chi^{2}(1)=\right.$ $2.09, p>.10]$. A $\chi^{2}$ test on the information-integration 
condition data was nonsignificant $\left[\chi^{2}(2)=1.49, p>.40\right]$, suggesting no difference in the proportion of learners across the control, long, and short feedback-processing time conditions.

Focusing only on the learners, we compared the trialsto-criterion measure across the control, long, and short feedback-processing time conditions separately for the rule-based and information integration tasks. These data are displayed in Figure 4B. For the rule-based category structure, the effect of condition was marginally significant $\left[F(2,43)=2.97, M S_{\mathrm{e}}=3,864.78, p=.06\right]$. To isolate the locus of this effect, we conducted a series of $t$ tests. As we predicted, it took the observers significantly longer to learn the rule-based task in the short feedback-processing condition (177 trials) than in the long feedback-processing condition [121 trials; $t(24)=$ $1.98, p<.05$ ] or the control condition [125 trials; $t(28)=$ $2.12, p<.05]$. Trials to criterion did not differ across the control and long feedback-processing conditions $(t<1)$. Also as predicted, the trials to criterion did not differ across the control (115 trials), long (130 trials), and short (128 trials) feedback-processing time conditions in the information integration task $(F<1)$. It is worth mentioning that the pattern of results observed for the proportion of learners/nonlearners and trials to criterion held across other window sizes (60 and 80) and accuracy criteria (.65 and .70), and thus is a robust effect.

We also applied a series of decision-bound models (see, e.g., Ashby, 1992) to the 80-trial learning window for all learners. No significant differences emerged across the control, long, and short feedback-processing time conditions, with the majority of observers in the rule-based conditions using rule-based strategies and the majority of observers in the information integration conditions using information integration strategies.

\section{GENERAL DISCUSSION}

These results add to the already substantial body of data suggesting that human category learning is mediated by functionally separate systems (e.g., Ashby et al., 1998; Ashby \& Ell, 2001). It is becoming increasingly unlikely that any single-system model will be able to account for the wide variety of category learning dissociations that have been reported. Even so, our goal was not so much to add to the abundant existing evidence in favor of multiple category learning systems. Rather, the present research was motivated by the more ambitious goal of discovering and studying key qualitative differences among these putative systems. We focused on the
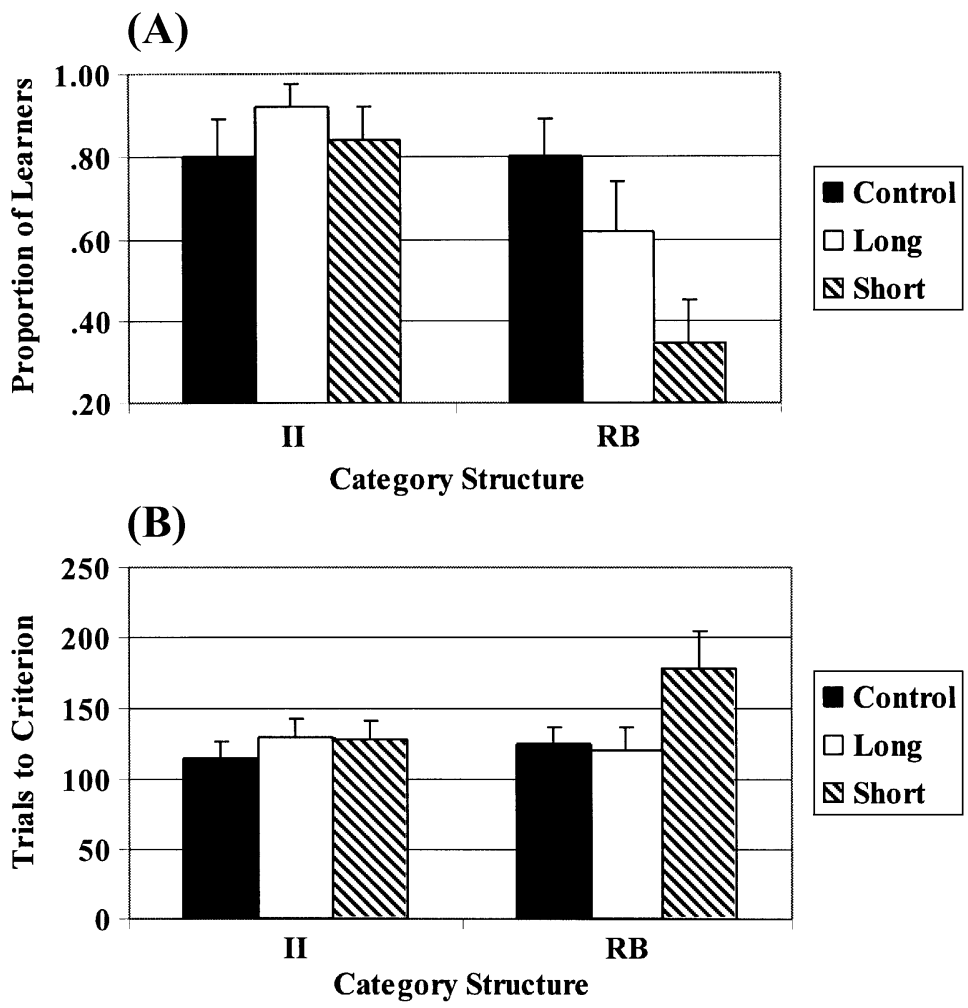

Figure 4. (A) Proportion of learners determined on the basis of a sliding 80-trial window and a $65 \%$ accuracy criterion. (B) Trials to criterion for the learners. II, information integration task; RB, rule-based task. 
two category learning systems hypothesized by the COVIS model (Ashby et al., 1998; Ashby \& Waldron, 1999). According to this model, rule-based category structures are learned by an explicit hypothesis-testing system that has full access to working memory and executive attention and learns through an active process of hypothesis generation and testing. In contrast, information-integration category learning is dominated by a procedural learning system that depends on reward-mediated learning and is largely implicit.

Even at this crude level of description, many strong a priori predictions can be made. The present study is the first to test the a priori prediction that attention and effort are required for the rule-based system to process the feedback signal fully, whereas feedback processing is essentially automatic in the procedural learning system. To test this prediction, we had observers perform a simple memory scanning task immediately following categorization (short feedback-processing time condition) or after a 2.5 -sec delay (long feedback-processing time condition). As we predicted, performance in the rule-based task was impaired in the short feedback-processing time condition relative to the long feedback-processing time condition. In particular, rule-based category learning was reduced, the proportion of observers who met a predefined performance criterion was reduced, and the number of trials necessary to reach the performance criterion was increased in the short feedback-processing time condition relative to the long feedback-processing time condition. On the other hand, no effect was observed in the information integration task, on either the proportion of observers meeting the predefined performance criterion or the number of trials necessary for the learners to reach criterion.

Our results, together with the dissociations reviewed in the introduction, are beginning to paint a fascinating picture of two very different category learning systems. The rule-based system is extremely flexible and can quickly make dramatic and abrupt changes in strategy. In particular, from one trial to the next it can reject one categorization rule and replace it with another rule that is vastly different from the first. The present study shows, however, that the cost of this flexibility is attention and effort - that is, the processes of rule selection, testing, rejection, and replacement place high demands on working memory and executive attention. In addition, the rule-based system is constrained to implementing categorization rules that are amenable to explicit reasoning. This feature makes it difficult or impossible for the rule-based system to perform well in our information integration conditions. In contrast, the procedural learning system appears to learn incrementally in a fashion that is heavily dependent on immediate feedback. Large changes in strategy will require many trials. Even so, this system has a number of advantages of its own. In particular, it can learn informationintegration category structures, and it processes the feedback signal automatically.

It is worth mentioning that the memory scanning task places considerable demands on verbal working memory. Baddeley (1986) argues for a verbal working memory store that is separate from a visuospatial working memory store. One interesting area for future research will be to examine the effects of visuospatial working memory tasks on rule-based and information-integration category learning. One possibility is that the rule-based system relies only on verbal working memory and that a visuospatial working memory task would not interfere with rule-based category learning. A second possibility is that either type of working memory task (verbal or visuospatial) will interfere with rule-based category learning. A third, intriguing possibility is that working memory is involved in both rule-based and informationintegration category learning, but that verbal working memory mediates rule-based learning, whereas visuospatial working memory mediates information integration learning. This represents a multiple-systems approach, but one that is different from COVIS. Clearly, more work is needed.

It should be stressed that the dissociation between the short and long feedback-processing time conditions and type of category structure reported in this article was predicted a priori by COVIS, and, furthermore, that these predictions were parameter free. It is hard to see how a single-system model could predict our results in a similar a priori fashion. Of course, it is possible that some single-system model could account for our results via post hoc manipulation of its parameters, but post hoc accounts are less parsimonious than parameter-free a priori predictions, regardless of the number of systems assumed by the two models.

\section{REFERENCES}

Ashby, F. G. (1992). Multidimensional models of categorization. In F. G. Ashby (Ed.), Multidimensional models of perception and cognition (pp. 449-484). Hillsdale, NJ: Erlbaum.

Ashby, F. G., Alfonso-Reese, L. A., Turken, A. U., \& Waldron, E. M. (1998). A neuropsychological theory of multiple systems in category learning. Psychological Review, 105, 442-481.

Ashby, F. G., \& ElL, S. W. (2001). The neurobiological basis of category learning. Trends in Cognitive Sciences, 5, 204-210.

Ashby, F. G., Ell, S. W., \& Waldron, E. M. (2003). Procedural learning in perceptual categorization. Memory \& Cognition, 31, 1114-1125.

Ashby, F. G., \& GotT, R. E. (1988). Decision rules in the perception and categorization of multidimensional stimuli. Journal of Experimental Psychology: Learning, Memory, \& Cognition, 14, 33-53.

AshbY, F. G., \& MadDox, W. T. (1998). Stimulus categorization. In M. H. Birnbaum (Ed.), Measurement, judgment, and decision making (pp. 251-301). New York: Academic Press.

Ashby, F. G., Maddox, W. T., \& BohIL, C. J. (2002). Observational versus feedback training in rule-based and information-integration category learning. Memory \& Cognition, 30, 666-677.

Ashby, F. G., Noble, S., Filoteo, J. V., WaldRon, E. M., \& Ell, S. W. (2003). Category learning deficits in Parkinson's disease. Neuropsychology, 17, 115-124.

Ashby, F. G., \& Waldron, E. M. (1999). The nature of implicit categorization. Psychonomic Bulletin \& Review, 6, 363-378.

BADDEley, A. D. (1986). Working memory. Oxford: Oxford University Press.

Brainard, D. H. (1997). Psychophysics software for use with MATLAB. Spatial Vision, 10, 433-436.

ERICKSON, M. A., \& KRUSCHKE, J. K. (1998). Rules and exemplars in category learning. Journal of Experimental Psychology: General, 127, 107-140.

HAZELTINe, E., \& Ivry, R. B. (2001). Motor skill. In V. S. Ramachan- 
dran (Ed.), Encyclopedia of the brain (pp. 183-200). San Diego: Academic Press.

Maddox, W. T., Ashby, F. G., \& Bohil, C. J. (2003). Delayed feedback effects on rule-based and information-integration category learning. Journal of Experimental Psychology: Learning, Memory, \& Cognition, 29, 650-662.

MADDOX, W. T., BoHIL, C. J., \& ING, A. D. (in press). Evidence for a procedural-learning based system in perceptual category learning. Psychonomic Bulletin \& Review.

Nosofsky, R. M., \& Johansen, M. K. (2000). Exemplar-based accounts of "multiple-system" phenomena in perceptual categorization. Psychonomic Bulletin \& Review, 7, 375-402.

PICKERING, A. D. (1997). New approache to study of amnesic patients: What can a neurofunctional philosophy and neural network methods offer? Memory, 5, 255-300.
Reber, P. J., \& SQuire, L. R. (1994). Parallel brain systems for learning with and without awareness. Learning \& Memory, 1, 217-229.

Schultz, W. (1992). Activity of dopamine neurons in the behaving primate. Seminars in Neuroscience, 4, 129-138.

Smith, E. E., Patalano, A., \& Jonides, J. (1998). Alternative strategies of categorization. Cognition, 65, 167-196.

WALDRON, E. M., \& AshBY, F. G. (2001). The effects of concurrent task interference on category learning: Evidence for multiple category learning systems. Psychonomic Bulletin \& Review, 8, 168-176.

Wickens, J. (1993). A theory of the striatum. New York: Pergamon.

Willingham, D. B. (1998). A neuropsychological theory of motor skill learning. Psychological Review, 105, 558-584.

Willingham, D. B., Nissen, M. J., \& Bullemer, P. (1989). On the development of procedural knowledge. Journal of Experimental Psychology: Learning, Memory, \& Cognition, 15, 1047-1060.

(Manuscript received May 30, 2003;

revision accepted for publication December 1, 2003.) 